\title{
DESENVOLVIMENTO DE NEOPLASIAS/ ADENOMAS AVANÇADOS COLORRETAIS NO SEGUIMENTO A LONGO PRAZO DE PACIENTES SUBMETIDOS A COLONOSCOPIA COM POLIPECTOMIA
}

\author{
Renata Andreoli ROSTIROLLA ${ }^{1}$, Júlio Carlos PEREIRA-LIMA², Cláudio Rolim TEIXEIRA ${ }^{3}$, \\ Aline Weyne SCHUCH${ }^{4}$, Camila PERAZZOLII e Carlos SAUL ${ }^{5}$
}

RESUMO - Contexto - A colonoscopia e a polipectomia diminuem a incidência do câncer colorretal, assim como a mortalidade dele decorrente. $\mathrm{O}$ intervalo efetivo entre os exames de seguimento é determinado por características clínicas e achados endoscópicos considerados como preditivos para o desenvolvimento de lesões neoplásicas colônicas avançadas. Objetivos - Avaliar a taxa de surgimento de lesões neoplásicas avançadas em pacientes submetidos a colonoscopias de seguimento em serviço de referência. Métodos - Foram incluídos 392 pacientes submetidos a dois ou mais exames colonoscópicos completos entre 1995 e 2005, constantes no banco de dados e que apresentavam, em pelo menos um dos exames, um adenoma colorretal. Foram analisados os dados da primeira e da última colonoscopias realizadas por cada paciente, ou daquela que mostrava lesão neoplásica avançada - considerado o desfecho principal do estudo. Os pacientes foram divididos de acordo com os achados endoscópicos do primeiro exame em grupos 1 ou de alto risco, 2 ou de baixo risco e 3 ou sem adenoma na colonoscopia inicial. Foram analisados e comparados entre os grupos o aparecimento de neoplasia colônica avançada e o tempo de seguimento até o desfecho. Resultados - Do total de pacientes, $27 \%$ apresentavam à colonoscopia inicial, adenomas avançados, 58,4\% lesões neoplásicas com displasia de baixo grau e $14,5 \%$ não apresentavam adenomas no exame inicial. A média etária foi de 59,54 $\pm 11,74$ anos. Vinte e seis vírgula quatro por cento das pessoas do grupo 1 apresentaram lesão neoplásica avançada ao longo do seguimento, enquanto tal desfecho ocorreu em 10,9\% e $5,3 \%$ dos pacientes nos grupos 2 e 3 , respectivamente $(P<0,05)$. O período médio de acompanhamento foi de 123,35 meses, sendo que o tempo médio entre o primeiro exame e aquele com o desfecho diferiu estatisticamente entre o grupo 1 e os demais, sendo de 104,02, 115,31 e 120,61 meses, respectivamente. Conclusões - Pacientes com neoplasia avançada à colonoscopia índice apresentam maior chance de possuir tais lesões, em relação aos demais grupos, durante o seguimento, e o surgimento de neoplasia avançada ocorre mais precocemente neste grupo.

DESCRITORES - Neoplasias colorretais. Adenoma. Pólipos intestinais. Colonoscopia. Programas de rastreamento.

\section{INTRODUÇÃO}

O câncer colorretal é a quarta neoplasia maligna mais frequente no Brasil e a terceira causa de morte por câncer ${ }^{(4)}$. O número de óbitos pela doença no nosso País supera o causado por câncer de colo uterino na população feminina ${ }^{(4)}$. Sua incidência é maior nas regiões mais ricas, particularmente nos estados do sudeste e sul ${ }^{(4,6)}$. O Ministério da Saúde considera a necessidade crescente de um programa de rastreamento para sua detecção precoce com vistas à diminuição dos índices de mortalidade.
A efetividade dos diferentes métodos de rastreamento foi analisada por força-tarefa do Serviço Preventivo dos Estados Unidos. Na ocasião foram estudados e comparados, em relação a custo-efetividade, os exames de sangue oculto nas fezes, retossigmoidoscopia flexível, a combinação dos anteriores, e à colonoscopia ${ }^{(10,12}$, 15, 17). Nos últimos anos, estudos comparativos têm enfatizado e indicado a colonoscopia como o exame inicial para o rastreamento do câncer colorretal devido sua melhor custo-efetividade em relação às outras opções de rastreamento, especialmente se considerados os níveis de adesão vislumbrados na

Trabalho realizado na Fundação Rio-grandense Universitária de Gastroenterologia (FUGAST) e no Curso de Pós-graduação em Ciências Médicas da Universidade Federal de Ciências da Saúde de Porto Alegre (UFCSPA)

Santa Casa de Porto Alegre; ${ }^{2}$ Departamento de Gastroenterologia da UFCSPA; ${ }^{3}$ FUGAST, Porto Alegre, RS.

Correspondência: Dr. Júlio Carlos Pereira-Lima - Rua Comendador Rheingantz, 910/801 - Bairro Bela Vista - 90450-020 - Porto Alegre, RS.

E-mail: jpereiralima@terra.com.br 
prática clínica - critério que influencia diretamente a relação custo-efetividade de qualquer exame com tal finalidade ${ }^{(15,17)}$. Desde então, a colonoscopia é tida como exame de eleição para rastreamento da população geral entre 50 e 60 anos de idade devido a sua elevada precisão diagnóstica e associado poder terapêutico.

Os adenomas colorretais - que se apresentam na forma de pólipos ou lesões planas - são as neoplasias mais frequentemente encontradas em colonoscopias de rastreamento, assim como em pacientes sintomáticos acima dos $50 \operatorname{anos}^{(3,19)}$. Devido ao conhecimento da sequência adenoma-carcinoma e das evidências científicas de que esta é a via principal da carcinogênese colorretal, é possível diminuir a incidência e a mortalidade da doença através de programas de rastreamento populacional $^{(5)}$.

Entre os anos de 1970 e 1990 era aceita a prática de colonoscopia anual a partir do diagnóstico de pólipo adenomatoso colorretal. A partir de estudos dos anos 90, a evolução dos adenomas passou a ser melhor compreendida e, atualmente, os pacientes são estratificados de acordo com o número, tamanho e histologia destas lesões pré-malignas diagnosticadas em exame chamado inicial e, a partir destes critérios, são recomendados para uma estratégia de vigilância endoscópica $^{(7,11,19)}$.

Dada a escassez de estudos de seguimento endoscópico, pretendeu-se avaliar a taxa de aparecimento de lesões adenomatosas colorretais avançadas em populações de alto e baixo risco, que se encontram sob vigilância endoscópica em serviço de referência do Rio Grande do Sul.

\section{MÉTODOS}

Foram incluídos no estudo 392 pacientes entre 19 e 99 anos de idade submetidos a dois ou mais exames colonoscópicos completos entre janeiro de 1995 e dezembro de 2005, constantes no banco de dados da Fundação Universitária Rio-grandense de Gastroenterologia (FUGAST), e que apresentaram em pelo menos um dos exames uma neoplasia colônica adenomatosa de qualquer tamanho (lesão polipóide ou plana). Todos os pólipos e lesões planas identificados foram removidos através de biopsia excisional com pinça fria ou "hot biopsy", ressecção com alça diatérmica ou mucosectomia em bloco ou por fatiamento ("piecemeal"). A FUGAST não atende pelo Sistema Único de Saúde e é serviço de referência para realização de procedimentos endoscópicos no sul do país. No serviço são realizadas atualmente cerca de 2.000 colonoscopia por ano.

Os exames foram realizados por três experimentados endoscopistas (com realização de mais de 5.000 exames cada) e a análise histológica das lesões ressecadas, realizada por patologista com especial interesse na área. A localização das neoplasias foi estimada usando marcos endoscópicos conhecidos e/ou através da medida do colonoscópio durante sua retirada a partir do ceco.

Todas as informações foram coletadas de um banco de dados informatizado, onde constavam os laudos endoscópicos, nome, sexo e data de nascimento dos pacientes, data e fotografias dos exames, resultados anatomopatológicos e todos os exames colonoscópicos anteriores e subsequentes ao período, realizados na instituição por cada paciente.

Foram excluídos do estudo os pacientes com diagnóstico endoscópico de doença inflamatória intestinal, polipose (mais de 100 pólipos) adenomatosa ou hiperplásica, e neoplasia maligna avançada diagnosticada no exame inicial, além dos pacientes com exames incompletos ou preparo de cólon considerado inadequado.

O estudo foi realizado nos moldes de uma coorte histórica, sendo os pacientes alocados em três grupos de acordo com os achados endoscópicos do exame índice como mostra a Tabela 1.

TABELA 1. Classificação dos grupos por achados endoscópicos à primeira colonoscopia

\begin{tabular}{lccc}
\hline Características & Grupo 1 & Grupo 2 & Grupo3 \\
\hline Número de adenomas (colonoscopia) & 3 ou mais & 1 ou 2 & 0 \\
Grau de displasia & alto & baixo ou nenhum & - \\
Dimensão do pólipo (cm) & $>$ que 1 & $<$ que 1 & - \\
Presença de histologia vilosa & Presença & Ausência & - \\
Presença de carcinoma in situ & Presença & Ausência & - \\
(pólipo malignizado) & & & \\
\hline
\end{tabular}

Todas as lesões neoplásicas identificadas foram removidas e analisadas histologicamente durante todo o período estudado. Para a classificação dos pacientes foi considerada a lesão histologicamente mais avançada de cada exame. O tamanho das lesões foi determinado, sempre que possível, através da medição direta da peça in vitro ou por comparação com a pinça de biopsia aberta (padrão de $8 \mathrm{~mm}$ ). Os pólipos de histologia inflamatória ou hiperplásicos não foram considerados neste estudo.

O desfecho principal foi o aparecimento de neoplasia colorretal avançada, definida como adenoma maior que $10 \mathrm{~mm}$, ou com histologia vilosa, ou displasia de alto grau, ou carcinoma - in situ ou invasivo. Além destes critérios, pacientes com três ou mais adenomas, independente da natureza histológica, foram considerados como apresentando o desfecho principal ou de alto risco. Lesões de menor relevância clínica e colonoscopias sem neoplasia adenomatosa foram consideradas desfechos secundários.

Os critérios avaliados e considerados para a estratificação de risco foram exclusivamente extraídos dos achados endoscópicos, não sendo considerados informações clínicas como indicação do exame, história familiar de pólipos ou de câncer colorretal.

Foi considerado como tempo de seguimento o intervalo entre a primeira e a última colonoscopia ou quando houvesse o surgimento do desfecho neoplasia avançada - quando o paciente era recenseado.

Os pacientes submetidos a colonoscopias com intervalo de até 6 meses após o exame inicial, tiveram os achados endoscópicos do segundo exame considerados como já existentes na colonoscopia índice e não como novas lesões neoplásicas. 
Os dados foram imputados em uma planilha do MS Excel e posteriormente analisados com recursos do pacote estatístico SPSS 13.0. As variáveis quantitativas foram apresentadas em forma de média e desvio-padrão e as qualitativas, através de frequência e percentual. Para a comparação de médias fez-se uso de análise de variância (ANOVA); para identificação de relação entre variáveis categóricas, qui ao quadrado de Pearson. Verificou-se a significância da relação das categorias pelos resíduos ajustados observados nas células. Foram confeccionadas curvas de sobrevida, pelo método Kaplan-Meier e estimadas as médias para os grupos, que foram comparadas por log rank. O nível de significância assumido foi de $5 \%$.

Este estudo foi aprovado pelo Comitê de Ética da FUGAST, vinculado ao Comitê de Ética em Pesquisa da Universidade Federal de Pelotas (UFPEL), RS.

\section{RESULTADOS}

Trezentos e noventa e dois pacientes foram incluídos no estudo. Destes, 121 eram homens $(30,9 \%)$ e 271 mulheres $(69,1 \%)$. A idade dos pacientes variou de 19 a 99 anos. A média foi de $59.54 \pm 11.74$ anos.

O número de colonoscopias estudadas foi de 1.232 (342 no grupo 1, 698 no grupo 2 e 192 no grupo 3), sendo 949 pólipos removidos e analisados histologicamente. Foram considerados os achados endoscópicos para análise estatística de 784 colonoscopias - considerando a primeira e a última de cada paciente ou a que apresentasse o desfecho principal.

De acordo com os achados endoscópicos do exame de base, os pacientes foram alocados em três grupos, conforme previamente mencionado. O grupo 1 foi composto por 106 pacientes que apresentavam adenomas com algum critério de gravidade como tamanho maior que $10 \mathrm{~mm}$, componente viloso, displasia de alto grau, em número de três ou mais, ou carcinoma em pólipo ou lesão plana (in situ ou invasivo) à colonoscopia inicial; do grupo 2 fizeram parte 229 indivíduos apresentando até dois adenomas tubulares menores que $10 \mathrm{~mm}$ e sem displasia de alto grau; o grupo 3 foi composto por 57 pacientes cuja primeira colonoscopia era negativa para lesões adenomatosas.

Dos 392 pacientes estudados, $26,4 \%(n=28)$ apresentaram na primeira colonoscopia adenomas avançados, preenchendo critérios de alto risco para desenvolvimento de carcinoma colorretal. A maioria dos indivíduos $(\mathrm{n}=229$ ou $58,4 \%)$ tinha lesões neoplásicas menores e/ou com histologia benigna, enquanto o menor grupo $(n=57$ ou $14,5 \%)$ não apresentava adenomas no exame inicial.

A média de idade (anos) dos pacientes dos grupos 1, 2 e 3 foi $61,27 \pm 11,25,59,01 \pm 11,29$ e $58,28 \pm 14,12$, respectivamente. Não houve diferença estatística entre as médias de idade, nem quanto ao número mediano de colonoscopias a que se submeteram os pacientes nos três diferentes grupos que foi igual a 3 (intervalo entre os quartis de 2,0 a 4,0 ).

Os resultados obtidos com a análise da evolução de cada grupo revelaram diferença estatística quanto ao aparecimento do desfecho (Tabela 2).
TABELA 2. Aparecimento de lesões neoplásicas nos diferentes grupos avaliados

\begin{tabular}{lcccc}
\hline \multirow{2}{*}{$\begin{array}{l}\text { Grupos } \\
\text { (número de pacientes) }\end{array}$} & $\begin{array}{c}\text { Evolução } \\
\text { Neoplasia } \\
\text { avançada }\end{array}$ & $\begin{array}{c}\text { Adenomas } \\
\text { pequenos }\end{array}$ & $\begin{array}{c}\text { Sem } \\
\text { neoplasia }\end{array}$ & Total \\
\hline 1-Alto risco & $28(26,4 \%)^{*}$ & $37(34,9 \%)$ & $41(38,6 \%)$ & 106 \\
2-Baixo risco & $25(10,9 \%)$ & $102(44,5 \%)$ & $102(44,5 \%)$ & 229 \\
3-Normal & $3(5,3 \%)$ & $31(54,4 \%) *$ & $23(40,4 \%)$ & 57 \\
Total & $56(14,3 \%)$ & $170(43,3 \%)$ & $166(42,3 \%)$ & 392 \\
\hline * Células estatisticamente significantes pelas análises dos resíduos ajustados & &
\end{tabular}

** Relação estatisticamente significante pelo qui ao quadrado de Pearson $(x=18,820, \mathrm{P}<0,05)$

De acordo com os resultados, $26,4 \%$ das pessoas que apresentavam neoplasia avançada no primeiro exame o fizeram novamente ao longo da vigilância endoscópica. No acompanhamento do segundo grupo, observou-se que apenas $10,9 \%$ dos indivíduos desenvolveram lesões de alto grau e que uma minoria $(5,3 \%)$ do grupo inicialmente sem adenomas foi flagrada apresentando lesões de relevância clínica ao longo do seguimento $(P<0,05)$.

Dez pacientes do grupo 1 , sendo $50 \%$ homens, apresentaram à colonoscopia inicial, lesões com diagnóstico histológico de carcinoma in situ. Destes, seis lesões eram planas e quatro de morfologia polipóide. Durante o seguimento dos pacientes foram diagnosticados mais dois casos de igual histologia, sendo os dois carcinomas identificados em espécimes de lesões planas, em ceco e cólon descendente. Ambos os pacientes, um de cada sexo, pertenciam inicialmente ao grupo 2 e apresentaram tal desfecho 47 e 7 meses após a colonoscopia inicial. Todos os 12 pacientes com diagnóstico de carcinoma in situ foram submetidos a tratamento endoscópico completo das lesões.

Dos 56 pacientes diagnosticados com neoplasia avançada ao longo do seguimento endoscópico, 28 pertenciam ao grupo 1,25 ao grupo 2 e apenas 3 eram oriundos do grupo 3. A Tabela 3 mostra a caracterização destas lesões identificadas na última colonoscopia, grupo por grupo.

TABELA 3. Caracterização por grupo das neoplasias avançadas diagnosticadas ao longo do seguimento endoscópico

\begin{tabular}{lcccc}
\hline Grupos & \multicolumn{2}{c}{ Características das neoplasias avançadas } & \\
& $>1 \mathrm{~cm}$, viloso, ou & Número igual ou & Carcinoma \\
displasia de alto grau & maior que 3 & Total & \\
\hline 1-Alto risco & 17 & 11 & 0 & 28 \\
2-Baixo risco & 19 & 4 & 2 & 25 \\
3-Normal & 3 & 0 & 0 & 3 \\
Total & 39 & 15 & 2 & 56 \\
\hline
\end{tabular}

O período médio de acompanhamento dos pacientes foi de 123,35 meses e o tempo de seguimento entre a primeira colonoscopia e o desfecho diferiu estatisticamente entre os grupos. Conforme a análise estatística e ilustrado na Tabela 4, o grupo 1 levou em média 104 meses para desenvolver o desfecho, enquanto os grupos 2 e 3 , considerados como de baixo risco, apresentaram período mais longo entre o exame inicial e o aparecimento do desfecho, estimado em 115,3 e 122,6 meses, respectivamente. 
TABELA 4. Tempo médio (meses) de seguimento dos grupos até o aparecimento de uma lesão avançada

\begin{tabular}{lcc}
\hline \multirow{2}{*}{ Grupos } & \multicolumn{2}{c}{ Sobrevida (meses) } \\
\cline { 2 - 3 } & Média & IC 95\% \\
\hline Alto risco & 104,02 & $88.45-119.59$ \\
Baixo risco & 115,31 & $108.03-122.58$ \\
Normal & 122,61 & $110.90-130.32$ \\
Total & 123,35 & $115,87-130,83$ \\
\hline
\end{tabular}

* Evento = lesão avançada;

Médias com diferençss estariscricamente significantes pelo mérodo de "log rank"

Na Figura 1, a curva de sobrevida de Kaplan-Meier demonstra que o grupo 1 apresenta maior aparecimento de neoplasia avançada em relação aos demais grupos ao longo do tempo $(P<0,05)$. Ou seja, pode-se afirmar que no grupo 1 surge mais rapidamente a neoplasia avançada que no grupo 2. Neste, entretanto, surge mais rapidamente a neoplasia avançada quando comparado ao grupo 3. Durante 2 anos, em 19,6\% dos pacientes do grupo 1, surgiu uma neoplasia avançada, enquanto que o grupo 2, 6,7\% dos pacientes apresentaram este desfecho. Já no grupo 3 não houve casos com esta evolução no mesmo período $(P<0,05)$.

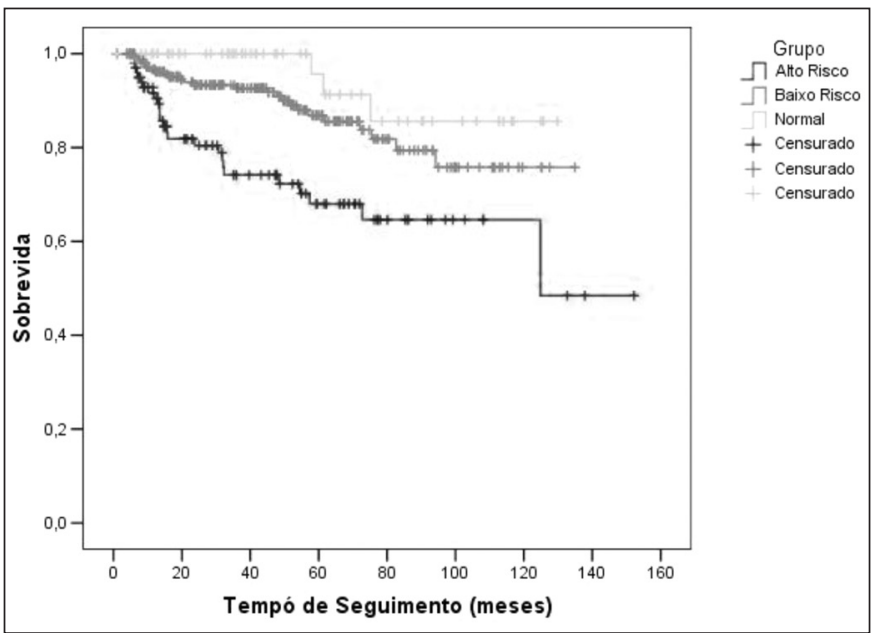

FIGURA 1. Curva de sobrevida acumulada de Kaplan-Meier expressando a evolução diferente entre os grupos durante o período de seguimento (meses)

\section{DISCUSSÃO}

O presente estudo avaliou o desenvolvimento de neoplasias colorretais avançadas em uma população de pacientes submetida a colonoscopia em serviço de referência no Rio Grande do Sul, estratificada em três grupos: alto risco, baixo risco e sem neoplasia no exame inicial. Além disto, foi determinado o tempo decorrido entre o primeiro exame e o aparecimento do desfecho nos diferentes grupos.

Os pacientes que apresentavam lesões adenomatosas avançadas no início do período em estudo tiveram maior percentagem $(P<0,05)$ de diagnóstico destas lesões ao longo do tempo (26.4\%) em comparação com os demais grupos $(10,9 \%$ e 5,3\%, respectivamente). Os resultados desta série corroboram os dados da literatura que elenca a multiplicidade de pólipos, o tamanho maior que $10 \mathrm{~mm}$, a presença de componente histológico viloso e o alto grau de displasia dos adenomas, como fatores preditivos positivos para a ocorrência de lesões avançadas e do carcinoma colorretal ${ }^{(11,19)}$.

É fato a escassez de estudos contemporâneos sobre rastreamento e seguimento de lesões neoplásicas colorretais. Quando recursos financeiros e humanos são disponíveis, a indicação da colonoscopia como exame inicial parece ser a mais adequada, já que representa o método diagnóstico considerado como padrão-ouro para detecção do câncer colorretal em todas as suas fases de evolução, bem como para sua prevenção, posto que possibilita a ressecção endoscópica das lesões precursoras ${ }^{(6)}$.

Ensaios clínicos randomizados e vários estudos de coorte mostraram que a colonoscopia com polipectomia reduz a incidência do câncer colorretal de $76 \%$ a $90 \%$, em comparação com o registro populacional geral ${ }^{(8,18)}$. Para minimizar a incidência e a mortalidade do câncer colorretal, pacientes com adenomas devem ser acompanhados em programas de vigilância endoscópica periódica para remoção de lesões sincrônicas não visualizadas inicialmente, assim como de adenomas metacrônicos e carcinomas ${ }^{(2,14)}$.

Os intervalos entre os exames de seguimento devem seguir as recomendações sugeridas pelos estudos populacionais e publicadas em guias atualizados, desenvolvidos pelas sociedades médicas afins. As características dos adenomas ressecados consideradas como preditivas para lesões avançadas futuras são: três ou mais adenomas, tamanho maior que $10 \mathrm{~mm}$, histologia vilosa e displasia de alto grau ${ }^{(1,8,16,19,21)}$. Atualmente sugerem-se para pacientes sem outros fatores de risco para câncer colorretal, exceto a presença de pólipos intestinais ressecados, as seguintes recomendações: (1) pacientes com pólipos hiperplásicos, especialmente retais, sejam considerados como tendo colonoscopia normal; (2) pacientes com até dois adenomas menores que $10 \mathrm{~mm}$ ou com displasia de baixo grau sejam reexaminados em 5 anos ou mais; (3) pacientes com três ou mais adenomas ou, pelo menos um deles maior de $10 \mathrm{~mm}$ ou com componente viloso ou displasia de alto grau, sejam submetidos a nova colonoscopia em 3 anos, desde que todos os pólipos tenham sido adequadamente removidos; (4) pacientes com mais de 10 pólipos devam repetir o exame num período inferior a 3 anos, de acordo com julgamento clínico, assim como devam ser considerados para possível síndrome familial subjacente, e (5) pacientes com pólipos sésseis removidos endoscopicamente por técnica de fatiamento devam fazer revisão colonoscópica em intervalos de 2 a 6 meses para verificar a remoção completa das lesões ${ }^{(19)}$.

Van STOLK et al. ${ }^{(16)}$ demonstraram que pacientes apresentando três ou mais adenomas na colonoscopia inicial tinham mais chance de apresentarem adenomas nos exames subseqüentes, quando comparados aos indivíduos inicialmente diagnosticados com até duas lesões.

A maioria dos estudos reporta que o tamanho do adenoma maior que $10 \mathrm{~mm}$ é fator preditivo de risco para lesões avançadas futuras. Alguns autores como ATKIN et 
al. ${ }^{(1)}$ e YANG et al. ${ }^{(21)}$ mostraram, através de estudos com retossigmoidoscopias, que pólipos adenomatosos volumosos (maiores que $10 \mathrm{~mm}$ ) apresentam associação positiva com o risco de neoplasia avançada durante a vigilância endoscópica.

Assim, como sugerem os resultados do presente estudo, muitas coortes observacionais demonstraram que a presença de componente viloso em adenomas ressecados durante exame de colonoscopia é fator preditivo para lesões avançadas nos exames que se seguem ${ }^{(19)}$.

A displasia de alto grau, quando presente, geralmente acontece em adenomas grandes e com componente viloso. LIEBERMAN et al. ${ }^{(11)}$ observaram que $10,9 \%$ dos doentes com adenomas com displasia de alto grau no início do seguimento, independente do tamanho da lesão, desenvolviam lesões avançadas num período de seguimento de 5 anos. A análise estatística dos dados da presente casuística mostrou que num período de 2 anos de seguimento, 19,6\% dos pacientes do grupo 1 apresentaram lesão neoplásica colorretal avançada, revelando assim, pior evolução deste grupo em comparação à literatura.

O estudo do grupo de Lieberman foi o primeiro grande estudo de seguimento a longo prazo de pacientes submetidos a colonoscopias de rastreamento ${ }^{(11)}$. Nessa casuística, a maior proporção de pacientes com adenomas durante o primeiro exame apresentava lesões tubulares menores que $10 \mathrm{~mm}$ e sem displasia de alto grau. Da mesma forma, verificou-se nesta casuística que $58,4 \%$ dos indivíduos apresentavam adenomas com estas características no exame inicial. Vale ressaltar, porém, que neste estudo não foram consideradas as indicações dos exames índices, diferentemente do trabalho prospectivo citado acima em que a indicação do exame era de rastreamento. Os autores concluíram que pacientes com até dois adenomas tubulares menores que $10 \mathrm{~mm}$ representam um grupo de baixo risco comparados com outros pacientes com neoplasia colônica ${ }^{(11)}$.

Neste estudo a média de idade não apresentou diferença estatística entre os grupos. Embora a quase totalidade dos trabalhos sobre seguimento colonoscópico em pacientes apresentando adenomas não relacione a idade como fator preditivo para desenvolvimento de lesão avançada, estudos japoneses encontraram esta relação e consideram a idade como fator de risco independente para tal desfecho ${ }^{(20)}$.

REX et al. ${ }^{(13)}$ publicaram em 1996, trabalho sobre o risco de pacientes com colonoscopias inicialmente normais desenvolverem adenomas após acompanhamento de 5 anos. Seus resultados revelaram que $27 \%$ dos indivíduos apresentaram pelo menos um adenoma no seguimento, porém apenas um paciente de 154 examinados desenvolveu adenoma maior que $10 \mathrm{~mm}$. Os autores consideraram o intervalo de 5 anos seguro para o reexame de pacientes inicialmente sem adenomas. Também no presente estudo, a maioria dos pacientes $(54,4 \%)$ do grupo com colonoscopia de base sem adenomas desenvolveu lesões de baixo grau ao longo do tempo. Entretanto, tal comparação não é possível, uma vez que os critérios de inclusão no presente estudo tenham selecionado pacientes com adenoma em pelo menos um dos exames colonoscópicos, sendo assim impossível estimar, com os dados que se têm, qual o risco de desenvolvimento de adenomas de uma população inicialmente sem a presença destas neoplasias.

Uma das críticas que poderia ser feita ao presente trabalho é sobre o delineamento retrospectivo, com coleta de informações em banco de dados. Porém, tal característica foi contrabalançada pelo expressivo número de pacientes inclú́dos no estudo, assim como pelo extenso período de 10 anos em que foram acompanhados. De fato, não há estudo semelhante prospectivo com seguimento de 10 anos. Caberia também a observação de que pacientes com adenomas avançados na colonoscopia índice tendem a ser submetidos a maior número de colonoscopias e em menor tempo que os demais grupos, o que poderia criar um fator de confusão, favorecendo o diagnóstico de mais lesões em menores intervalos de tempo no grupo 1, influenciando assim, nos resultados obtidos. Entretanto, a mediana de colonoscopias a que os pacientes foram submetidos foi a mesma (3) nos três grupos examinados, o que anula este possível viés. Outro fator relevante é que informações clínicas como história familiar de câncer colorretal ou de adenomas, indicação do exame e presença ou não de sintomas, não foram consideradas, embora sejam aspectos que reconhecidamente afetam o desenvolvimento do desfecho em questão. Todavia, o objetivo deste estudo foi avaliar exclusivamente os achados endoscópicos em colonoscopias consideradas de base e, a partir deles, analisar sua relação com o surgimento de lesão colorretal avançada.

$\mathrm{O}$ estudo dos exames de colonoscopia realizados na população rio-grandense com diagnóstico de adenoma e sob vigilância endoscópica permite concluir que os pacientes com adenomas avançados, diagnosticados à primeira colonoscopia, têm maior chance de apresentarem tais lesões no decorrer do seguimento endoscópico, quando comparados àqueles oriundos de grupos de menor risco. Além disto, o tempo médio para o aparecimento de lesões avançadas também é menor no grupo de alto risco, em comparação aos demais, corroborando a necessidade de vigilância endoscópica mais agressiva nessa população. 
Rostirolla RA, Pereira-Lima JC, Teixeira CR, Schuch AW, Perazzoli C, Saul C. Development of colorectal advanced neoplasia/adenomas in the long-term follow-up of patients submitted to colonoscopy with polipectomy. Arq Gastroenterol. 2009;46(2):167-72.

ABSTRACT - Context - Colonoscopy with polypectomy reduces the incidence of colorectal cancer and its associated mortality. The ideal interval between surveillance examinations is determined by clinical features and endoscopic findings considered as risk factors to the development of advanced colonic neoplasias. Objective - To determine the development rate of advanced neoplasia in patients submitted to surveillance colonoscopy in a tertiary referral center. Methods - Three hundred and ninety two patients who underwent two or more complete colonoscopies between 1995 and 2005 , and who have at least one diagnosed colorectal adenoma entered into the study. The endoscopic findings of the first and subsequent colonoscopies of each patient were analysed, considering advanced neoplasia as the main outcome. The patients enrolled were divided in accordance to the first colonoscopy findings in groups 1 or high risk; 2 or low risk; and 3 or without adenoma at the first colonoscopy. The development of advanced colorectal neoplasia and the period of surveillance until the outcome were analysed and compared among groups. Results - Twenty eight per cent of patients had advanced adenomas at index colonoscopy; $57.8 \%$ presented with low grade dysplasia neoplastic lesions and $14.1 \%$ had no adenoma at the first examination. The mean age was $59.54 \pm 11.74$ years. Twenty six point four per cent of subjects from group 1 presented with advanced neoplasia during the surveillance period, while this outcome occurred in $10.9 \%$ and $5.3 \%$ of patients from groups 2 and 3 , respectively $(P<0,05)$. The mean period of surveillance was 123.35 months, and the mean time between the first examination and the one which presented with the outcome statistically differed among group 1 and the others, being 104.02, 115.31 and 120.61 months, respectively. Conclusions - Patients with advanced neoplasia at index colonoscopy presented with a higher probability of harbouring this condition during the follow-up when compared with other two groups. These lesions also occur earlier in this patients than in the ones without these lesions at the first examination.

HEADINGS - Colorectal neoplasms. Adenoma. Intestinal polyps. Colonoscopy. Mass screening.

\section{REFERÊNCIAS}

1. Atkin WS, Morson BC, Cuzick J. Long-term risk of colorectal cancer after excision of rectosigmoid adenomas. N Engl J Med. 1992;326:658-62.

2. Becker, F, Nusko G, Welke J, Hahn EG, Mansmann U. Follow-up after colorectal polypectomy: a benefit-risk analysis of German surveillance recommendations. Int J Colorectal Dis. 2007;22:929-39.

3. Binda V, Pereira-Lima J, Nunes CA, Falkemberg LT, Azambuja DB, Cruz JV Is there a role for sigmoidoscopy in symptomatic patients? Analysis of a study correlating distal and proximal colonic neoplasias detected by colonoscopy in a symptomatic population. Arq Gastroenterol. 2007;44:2-7.

4. Brasil. Ministério da Saúde. Secretaria de Atenção à Saúde. Estimativas 2008: incidência de câncer no Brasil. Rio de Janeiro: INCA; 2007.

5. Chen CD, Yen MF, Wang WM, Wong JM, Chen TH. A case-cohort study for the disease natural history of adenoma-carcinoma and de novo carcinoma and surveillance of colon and rectum after polypectomy: implication for efficacy of colonoscopy. Br J Cancer. 2003;88:1866-73.

6. Coelho JCCGP, Savi D, Coelho JRGP, Araujo SEA, Habr-Gama A, GamaRodrigues J. Papel da colonoscopia com magnificação de imagem associada à cromoscopia no diagnóstico diferencial entre lesões neoplásticas e não-neoplásticas do intestino grosso. ABCD Arq Bras Cir Dig. 2005;18:64-70.

7. Colquhoun P, Chen HC, Kim JL, Efron J, Weiss EG, Nogueras JJ, Vernava AM, Wexner SD. High compliance rates observed for follow up colonoscopy post polypectomy are achievable outside of clinical trials: efficacy of polypectomy is not reduced by low compliance for follow up. Colorectal Dis. 2004;6:158-61

8. Davila RE, Rajan E, Baron TH, Adler DG, Egan JV, Faigel DO, Gan SI, Hirota WK, Leighton JA, Lichtenstein D, Qureshi WA, Shen B, Zuckerman MJ, VanGuilder T, Fanelli RD; Standards of Practice Committee, American Society for Gastrointestinal Endoscopy. ASGE guideline: colorectal cancer screening and surveillance. Gastrointest Endosc. 2006;63:546-57.

9. Dawson B, Trapp R. Basic and clinical biostatistcs. 3rd edition. New York: Lange Medical Books/McGraw-Hill; 2001.

10. Frazier AL, Colditz GA, Fuchs CS, Kuntz KM. Cost-effectiveness of screening for colorectal cancer in the general population. JAMA. 2000;254:1954-61.

11. Lieberman D, Weiss DG, Harford WV, Ahnen DJ, Provenzale D, Sontag SJ, Schnel TG, Chejfec G, Campbell DR, Kidao J, Bond JH, Nelson DB, Triadafilopoulos G, Ramirez FC, Collins JF, Johnston TK, McQuaid KR, Garewal H, Sampliner RE, Esquivel R, Robertson D. Five-year colon surveillance after screening colonoscopy. Gastroenterology. 2007;133:1077-85.
12. O’Leary BA, Olynyk JK, Neville AM, Plattlel CF. Cost-effectiveness of colorectal cancer screening: comparison of community-based flexible sigmoidoscopy with fecal occult blood testing and colonoscopy. J Gastroenterol Hepatol. 2004;19:38-47.

13. Rex DK, Cummings OW, Helper DJ, Nowak TV, McGill JM, Chiao GZ, Kwo PY, Gottlieb KT, Ikenberry SO, Gress FG, Lehman GA, Born LJ. 5-year incidence of adenomas after negative colonoscopy in asymptomatic average-risk persons. Gastroenterology. 1996;111:1178-81.

14. Robertson DJ, Greenberg ER, Beach M, Sandler RS, Ahnen D, Haile RW, Burke CA, Snover DC, Bresalier RS, McKeown-Eyssen G, Mandel JS, Bond JH, Van Stolk RU, Summers RW, Rothstein R, Church TR, Cole BF, Byers T, Mott L, Baron JA. Colorectal cancer in patients under close colonoscopic surveillance. Gastroenterology. 2005; 129:34-41.

15. Sonnenberg A, Delco F, Inadomi JM. Cost-effectiveness of colonoscopy in screening for colorectal cancer. Ann Intern Med. 2000;133:573-84.

16. van Stolk RU, Beck GJ, Baron JA, Haile R, Summers R. Adenoma characteristics at first colonoscopy as predictors of adenoma recurrence and characteristics at follow-up. The Polyp Prevention Study Group. Gastroenterology. 1998;115:13-8.

17. Vijan S, Hwang EW, Hofer TP, Hayward RA. Which colon cancer screening test? A comparison of costs, effectiveness, and compliance. Am J Med. 2001;111:593601.

18. Winawer SJ, Zauber AG, Ho MN, O’Brien MJ, Gottlieb LS, Sternberg SS, Waye JD, Schapiro M, Bond JH, Panish JF, et al. Prevention of colorectal cancer by colonoscopic polypectomy. The National Polyp Study Workgroup. N Engl J Med 1993;329:1977-81.

19. Winawer SJ, Zauber AG, Fletcher RH, Stillman JS, O'Brien MJ, Levin B, Smith RA, Lieberman DA, Burt RW, Levin TR, Bond JH, Brooks D, Byers T, Hyman N, Kirk L, Thorson A, Simmang C, Johnson D, Rex DK. Guidelines for colonoscopy surveillance after polypectomy: a consensus update by the US Multi-Society Task Force on Colorectal Cancer and the American Cancer Society. CA Cancer J Clin. 2006;56:143-59.

20. Yamaji Y, Mitsushima T, Ikuma H, Watabe H, Okamoto M, Kawabe T, Wada $\mathrm{R}$, Doi H, Omata M. Incidence and recurrence rates of colorectal adenomas estimates by annually repeated colonoscopies on asymptomatic Japanese. Gut. 2004:53:568-72.

21. Yang G, Zheng W, Sun QR, Shu XO, Li WD, Yu H, Shen GF, Shen YZ, Potter JD, Zheng S. Pathologic features of initial adenomas as predictors for metachronous adenomas of the rectum. J Natl Cancer Inst. 1998;90:1661-5.

Recebido em 7/7/2008

Aprovado em 3/10/2008 